\title{
RESPUESTA A LA CARTA AL DIRECTOR TITULADA: "REFERENCIA AL ARTÍCULO: ANÁLISIS MULTIVARIADO DE RECIDIVA Y PROGRESIÓN EN EL CARCINOMA DE CÉLULAS TRANSICIONALES DE VEJIGA EN ESTADIO T1. VALOR PRONÓSTICO DE P53 Y KI67"
}

\author{
A. RODRÍGUEZ ALONSO*, S. PITA FERNÁNDEZ** \\ *Servicio de Urología. Hospital Arquitecto Marcide. El Ferrol. La Coruña. \\ **Unidad de Epidemiología Clínica y Bioestadística. Hospital Juan Canalejo. La Coruña.
}

Actas Urol Esp. 27 (9): 746-747, 2003

\section{Estimado Director:}

En relación al estudio realizado por los autores de esta carta, publicado en Actas Urológicas Españolas en febrero de $2003^{1}$, sobre el cual se publica una Carta al Director en el número de junio de $2003^{2}$, nos gustaría realizar una serie de aclaraciones, que exponemos a continuación.

Con respecto al comentario sobre las 177 variables analizadas y sobre el hecho de que el azar, por sí mismo, puede concluir un gran número de variables con significación estadísti$\mathrm{ca}^{2}$, nos gustaría indicar que, efectivamente, se introdujeron 177 variables por cada uno de los pacientes incluidos en el estudio, y antes de efectuar el análisis multivariado, se procedió de la siguiente forma: en primer lugar se realizó un análisis univariado de todas ellas, en relación con las variables respuesta (recidiva y progresión). Posteriormente, se introdujeron en el análisis de regresión múltiple aquellas variables que resultaron estadísticamente significativas en el análisis univariado y las variables clínicamente relevantes.

Para evitar incluso el posible efecto del azar, que señalan los autores de la Carta al Director ${ }^{2}$, en el análisis univariado consideramos sólo como significativos los valores de $\mathrm{p}<0,01$, con el objetivo de minimizar el error de tipo I. El hecho de utilizar, por otra parte, variables clínicamente relevantes (con valores de $\mathrm{p}>0,01$ ), permite que el sentido clínico sea el que dirija el análisis estadístico, para no convertir el estudio en un "fishing expedition".

En relación al hecho de que no se refieran las indicaciones y grupos de tratamiento y que no se analice la repercusión del tratamiento sobre la evolución y los resultados ${ }^{2}$, desearíamos indicar lo siguiente: existe evidencia científica suficiente de que la quimio o inmunoterapia intravesical adyuvante a la resección transuretral de los tumores $\mathrm{T} 1$, pueden modificar la supervivencia libre de recidiva y probablemente la supervivencia libre de progresión, en el caso de la inmunoterapia.

No obstante, el estudio por nosotros realizado, que es un estudio de cohortes, con seguimiento retrospectivo y prospectivo, no ha sido diseñado para evaluar la eficacia terapéutica de diferentes intervenciones, ya que para ello sería preciso realizar un ensayo clínico aleatorizado. La inclusión de variables terapéuticas que pueden modificar el pronóstico, en un estudio no aleatorizado, conlleva la introducción de sesgos de selección. Es por ello que no se ha analizado la repercusión de dichos tratamientos sobre la evolución y resultados obtenidos.

En los que se refiere a la explicación de los autores de la Carta al Director sobre el menor Riesgo Relativo de los tumores G2 y G3, con respecto a $\operatorname{los} G 1^{2}$, queremos apuntar que las diferencias en el riesgo de recidiva de los G2 y G3, 
con respecto a los G1, no fueron en ningún caso estadísticamente significativas $(p=0,1134 \quad y$ $\mathrm{p}=0,0833$, respectivamente). Por lo tanto cualquier conclusión extraída, estaría basada en un resultado no significativo.

Por lo que respecta a la relación del patrón de crecimiento endoscópico y el grado histológico, con la progresión tumoral ${ }^{2}$, nos gustaría señalar que en nuestro estudio, la morfología endoscópica sólida del tumor no modificó significativamente la progresión, en el análisis multivariado, aunque sí lo hizo en el análisis univariado (Log rank=4,08; $\mathrm{p}=0,0435$ ), como se puede observar en el texto y en la Tabla IV del trabajo original ${ }^{1}$. Sin embargo, el patrón de crecimiento microscópico del tumor, que constituye una variable con menor carga de subjetividad que su aspecto endoscópico, se mostró como una variable independiente de progresión $(\mathrm{RR}=55,19)$, en nuestro estudio.

En relación al papel del grado histológico en la progresión de los tumores de la categoría T1, opinamos que la importancia de esta variable en la progresión tumoral es cuando menos controvertida, ya que a pesar de que encontramos estudios en los que se demuestra una asociación, existen muchos estudios multivariados en los que el grado histológico no se muestra como una variable independiente de progresión tumoral ${ }^{3-8}$.

Por último, en relación a la inclusión de la variable "recidiva temprana" ${ }^{2}$, queremos indicar que en nuestro estudio se analizaron diversos aspectos de la recidiva (número de recidivas, existencia de recidiva en la cistoscopia efectuada a los 3 meses de la primera resección transuretral, entre otros), sin embargo no se observó una asociación estadísticamente significativa con la progresión en el análisis univariado, por lo que se decidió no incluir estas variables en el análisis multivariado.

\section{REFERENCIAS}

1. RODRÍGUEZ ALONSO A, PITA FERNÁNDEZ S, GONZÁLEZ-CARRERÓ J, NOGUEIRA MARCH JL.: Análisis multivariado de recidiva y progresión en el carcinoma de células transicionales de vejiga en estadio T1. Valor pronóstico de p53 y ki67. Actas Urol Esp 2003; 27 (2): 132-141.

2. PALOU REDORTA J, MILLÁN RODRÍGUEZ F.: Referencia al artículo: análisis multivariado de recidiva y progresión en el carcinoma de células transicionales de vejiga en estadio $\mathrm{T} 1$. Valor pronóstico de p53 y ki67. Actas Urol Esp 2003; 27 (6): 478-478.

3. LIUKKONEN T, RAJALA P, RAITANEN M, RINTALA E, KAASINEN E, LIPPONEN P.: Prognostic value of MIB-1 score, p53, EGFr, mitotic index and papillary status in primary superficial (Stage pTa/T1) bladder cancer: a prospective comparative study. Eur Urol 1999; 36: 393-400.

4. ZLOTTA AR, NOEL JC, FAYT I et al.: Correlation and prognostic significance of p53, p2 $1^{\mathrm{WAFl} / \mathrm{CIPl}}$ and ki67 expression in patients with superficial bladder tumours treated with Bacillus Calmette-Guérin intravesical therapy. $J$ Urol 1999; 161: 792-798.

5. HERR HW, BADALAMENT RA, AMATO DA, LAUDONE VP, FAIR WR, WHITMORE WF JR.: Superficial bladder cancer treated with Bacillus CalmetteGuérin: a multivariate analysis of factors affecting tumor progression. J Urol 1989; 141: 22-29.

6. SCHMITZ-DRÄGER BJ, KUSHIMA M, GOEBELL P et al.: p53 and MDM2 in the development and progression of bladder cancer. Eur Urol 1997; 32: 487-493.

7. SERTH J, KUCZYK MA, BOKEMEYER C et al.: p53 immunohistochemistry as an independent prognostic factor for superficial cell carcinoma of the bladder. Br J Cancer 1995; 71: 201-205.

8. LACOMBE L, DALBAGNI G, ZHANG ZF et al.: Overexpression of p53 protein in a high-risk population of patients with superficial bladder cancer before and after Bacillus Calmette-Guérin therapy: correlation to clinical outcome. J Clin Oncol 1996; 14: 2646-2652.

Dr. A. Rodríguez Alonso

Servicio de Urología

Hospital Arquitecto Marcide

Ctra. San Pedro de Leixa, s/n

15405 El Ferrol (La Coruña)

(Trabajo recibido el 29 julio de 2003) 